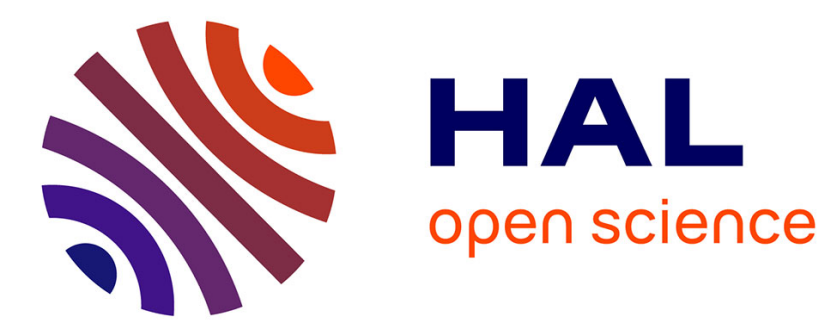

\title{
RÉPONSES ÉCHOGRAPHIQUES DE CIBLES INSONIFIÉES PAR DES TRADUCTEURS LARGE BANDE À PROFIL NON-UNIFORME
}

\author{
A. Lhémery, D. de Vadder
}

\section{- To cite this version:}

A. Lhémery, D. de Vadder. RÉPONSES ÉCHOGRAPHIQUES DE CIBLES INSONIFIÉES PAR DES TRADUCTEURS LARGE BANDE À PROFIL NON-UNIFORME. Journal de Physique IV Proceedings, 1992, 02 (C1), pp.C1-827-C1-830. 10.1051/jp4:19921180 . jpa-00251142

HAL Id: jpa-00251142

https://hal.science/jpa-00251142

Submitted on 1 Jan 1992

HAL is a multi-disciplinary open access archive for the deposit and dissemination of scientific research documents, whether they are published or not. The documents may come from teaching and research institutions in France or abroad, or from public or private research centers.
L'archive ouverte pluridisciplinaire HAL, est destinée au dépôt et à la diffusion de documents scientifiques de niveau recherche, publiés ou non, émanant des établissements d'enseignement et de recherche français ou étrangers, des laboratoires publics ou privés. 


\title{
RÉPONSES ECHOGRAPHIQUES DE CIBLES INSONIFIEES PAR DES TRADUCTEURS LARGE BANDE À PROFIL NON-UNIFORME
}

\author{
A. LHÉMERY et D. de VADDER \\ Laboratoire MSS/MAT URA 850 C.N.R.S., Equipe Ultrasons, Ecole Centrale de Paris, Grande Voie \\ des Vignes, F-92295 Châtenay-Malabry cedex, France
}

\begin{abstract}
Résumé - Les traducteurs classiquement utilisés en contrôle non-destructif des matériaux (CND) vibrent approximativement en piston avec un profil uniforme de source. En échographie bande étroite, la modification de ce profil (apodisation) est un moyen connu pour diminuer l'amplitude des lobes secondaires de diffraction. Nous montrons ici l'intérêt de telles modifications dans le cas d'imagerie large bande en étudiant la réponse échographique de cibles immergées de forme simple. La simplification de la structure spatio-temporelle du champ insonifiant la cible entraîne une simplification de cette réponse. Un modele récent est utilisé pour faire une étude paramétrique de cette influence.

Abstract - Commonly used transducers in nondestructive testing (CND in french initials) approximately vibrate as piston with an uniform amplitude distribution. In narrowband imaging, a well-known mean of avoiding diffraction secondary lobes consists in changing the shape of this distribution. Here we show the advantages of such a change in broadband imaging. For this, we study echo-responses from immersed simple shaped targets for different amplitude distributions. Simplification of time and space dependence of the incident field leads to this of echo-responses. A recently developed modeling is used for a parametric study of this effect.
\end{abstract}

\section{Modélisations des réponses échographiques.}

La réponse impulsionnelle acoustique du problème complet émission / diffusion / réception est donnée sous la forme de deux intégrales explicites, limitées à la surface insonifiée de la cible $(T)$ [1,2], liant la vitesse particulaire $v(t)$ à la surface émettrice à la moyenne instantanée de la pression $\langle p\rangle(t)$ appliquée à la surface réceptrice. Si l'on s'intéresse au cas de cibles immergées d'impédance spécifique très grande devant celle du milieu de propagation $\rho_{0} c$, la forme générale donnée dans $[1,2]$ se simplifie en une seule intégrale,

$$
<p>(t)=v(t) * H(t), \text { où }, \quad H(t)=\iint_{T}^{\alpha} \frac{\alpha\left(r_{T}\right)}{2} \frac{\cos \theta_{C-T}^{\prime}}{\rho_{0} c} h^{\partial t}\left(r_{T}, t\right) * h^{\partial t}\left(r_{T}, t\right) d S_{T} .
$$

$\alpha(\mathbf{r})=4 \pi / \Omega(\mathbf{r})$ où $\Omega(\mathbf{r})$ est l'angle solide du milieu de propagation vu depuis le point $\mathbf{r}$. L'angle $\theta^{\prime}{ }_{\mathrm{C}-\mathrm{T}}$ est formé par la normale au point courant avec le centre du traducteur. L'émission transitoire et par réciprocité la réception par le traducteur fonctionnant en piston, sont incluses dans (1) sous la forme de l'autoconvolution de $h^{\partial t}(r, t)$, réponse impulsionnelle en pression à l'émission. $h^{\partial t}(r, t)$ est définie par,

$$
h^{\partial t}(r, t) \equiv \rho_{0} \partial / \partial t \iint_{R} \Gamma\left(r_{R}\right) \frac{\delta\left(t-\left|r_{R}-r\right| / c\right)}{2 \pi\left|r_{R}-r\right|} d S_{R},
$$

où $R$ est la surface active du traducteur. $\Gamma(\mathbf{r})$ est la dépendance spatiale de la source de vitesse particulaire; cette fonction est supposée être aussi la fonction de sensibilité à la pression du traducteur en réception. Pour les traducteurs disque (plan ou focalisé), ou rectangulaire, $h^{\partial t}(r, t)$ est connue analytiquement si $\Gamma(r)$ est uniforme. Sinon, le principe de superposition permet de la calculer par sommation des réponses de traducteurs uniformes de rayons appropriés [3].

\section{Champ transitoire rayonné par un traducteur circulaire plan, uniforme ou non.}

Pour les points situés sur l'axe $(\rho=0, z)$ d'un traducteur disque, du fait de la symétrie cylindrique, la pression impulsionnelle rayonnée (2) est proportionnelle à la dérivée du profil de source, a un changement de variable non-linéaire près [3,4]. Ainsi, l'expression (2) devient dans le cas du disque plan, 


$$
\mathrm{h}^{\partial \mathrm{t}}(0, \mathrm{z} ; \mathrm{t})=\rho_{0} \mathrm{c} \partial / \partial \mathrm{t} \Gamma\left[\sqrt{(\mathrm{ct})^{2}-\mathrm{z}^{2}}\right], \mathrm{z}<\mathrm{ct}<\sqrt{\mathrm{z}^{2}+\mathrm{a}^{2}},
$$

' $a$ ', rayon du traducteur. Pour le traducteur uniforme $\Gamma\left(\rho_{R}\right)=1, \rho_{R}<a$, on a l'expression remarquable,

$$
h^{\partial t}(0, z ; t)=\rho_{0} c \partial / \partial t\left[\Pi\left(z / c, \sqrt{z^{2}+a^{2}} / c\right)\right]=\rho_{0} c\left[\delta(t-z / c)-\delta\left(t-\sqrt{z^{2}+a^{2}} / c\right)\right],
$$

$\Pi(.,$.$) , fonction "porte". Le \delta$ de Dirac de surpression à l'instant $t=z / c$ correspond à l'arrivée d'une onde dite 'plane', celui de dépression à l'instant $t=\left[\left(\mathrm{z}^{2}+\mathrm{a}^{2}\right)^{1 / 2}\right] / \mathrm{c}$ à celle d'une onde dite 'de bord'.

Pour les points situés en dehors de l'axe, l'onde plane arrivant à $t=z / c$ existe tant que $\rho<a$. Les contributions venant du bord du traducteur n'arrivent plus toutes au même instant, la symétrie étant rompue, mais entre les instants $\left[\left[z^{2}+(a-\rho)^{2}\right]^{1 / 2}\right] / c$ et $\left[\left[z^{2}+(a+\rho)^{2}\right]^{1 / 2}\right] / c$, sous la forme d'une dépression quand $\rho<a$ (maximale pour les instants extrêmes) ou d'une surpression puis d'une dépression quand $\rho>a$.

La discontinuité du profil au bord du traducteur est à l'origine du fait que l'amplitude de l'onde de bord sur l'axe est égale et opposée à celle de l'onde plane. Si le profil est adouci de sorte que l'amplitude vibratoire tend vers zéro de manière continue quand on s'approche du bord du traducteur (resp. du centre), l'onde de bord (resp. plane) n'est plus localisée en temps et son amplitude est moindre. On peut parler alors de traducteur 'à onde plane' (resp. 'à onde de bord'). Cette diminution d'amplitude existe pour tous les points du champ mais ne se traduit pas mathématiquement aussi simplement que pour les points de l'axe [3].

En imagerie bande étroite, les lobes de diffraction sont fortement diminués par l'apodisation de la source. Physiquement, la disparition des lobes de diffraction et la diminution de la contribution des ondes de bord sont deux manifestations du même phénomène.

La modification du profil de source va surtout simplifier la structure spatio-temporelle du champ près du traducteur où les contributions planes et de bord peuvent être séparées temporellement, même après convolution avec $v(t)$; seule l'une ou l'autre de ces contributions sera d'amplitude significative. Loin du traducteur, la structure spatio-temporelle est plus simple, quelle que soit la répartition de source; l'avantage d'une source à profil non-uniforme est alors moindre a priori. Notons que les termes "près" et "loin" dépendent fortement de $\mathrm{v}(\mathrm{t})$, elle-même directement liée à la réponse électro-acoustique du traducteur.

Différents procédés de fabrication ont été mis en œuvre pour modifier le profil de vitesse tout en conservant un fonctionnement en mode piston pour des matériels à bande étroite. A notre connaissance, seuls Brittain et Weight [5] ont appliqué de telles transformations à l'imagerie large bande spécifiquement, notamment en privilégiant les ondes de bord; leur méthode de fabrication consiste lors de la polarisation initiale de l'élément piezoélectrique à appliquer à des anneaux concentriques des tensions différentes. Aucun traducteur large bande à profil modifié ne semble à l'heure actuelle commercialement disponible. Dans la suite, nous montrons par simulation l'intérêt de tels systèmes en échographie large bande (CND, médicale etc...).

\section{Réponses de cibles insonifiées par des traducteurs de différents profils.}

En simplifiant le champ incident, on simplifie la structure des échos renvoyés par les cibles reçus par le traducteur, d'autant plus que, par réciprocité, les effets de diffraction liés à la réception sont diminués identiquement à ceux de l'émission. La suite de l'étude met en évidence l'importance de cet effet.

Nous étudions trois traducteurs de même diamètre, le premier uniforme, le deuxième à onde plane (TOP) et le troisième à onde de bord (TOB). Leurs profils sont présentés Fig.1. Le traducteur utilisé dans les expériences (Panametrics V3289, $19 \mathrm{~mm} \emptyset$ ) se comporte comme un piston uniforme [6]; les traducteurs TOP ou TOB n'ont d'existence que théorique. La comparaison "simulation / expérience" porte donc sur les résultats pour le traducteur uniforme. L'énergie rayonnée par les traducteurs à profil non-uniforme sera moindre que celle rayonnée par celui uniforme. Ce problème est technologique: pour le traducteur uniforme, la polarisation globale de l'élément piezoélectrique est orientée pour maximiser le transfert électromécanique; quelle que soit la méthode choisie pour modifier le profil de source, elle fera appel à une polarisation partielle de l'élément piezoélectrique dont le rendement ne pourra être qu'inférieur. Cependant, comme nous le verrons, l'énergie supérieure du traducteur uniforme est à l'occasion portée par des échos qui rendent plus délicate l'interprétation des signaux reçus.

Les cibles étudiées sont un disque de $4 \mathrm{~mm} \varnothing$ et un cône de $150^{\circ}$ d'angle au sommet et de même diamètre maximal; fabriquées en laiton, elles répondent au critère de grande impédance vis à vis du milieu de propagation (eau). Elles sont placées à 35 puis $120 \mathrm{~mm}$ du traducteur, centrées sur son axe, la normale au disque étant confondue avec l'axe du traducteur. La dépendance temporelle de la source est proche d'un cycle unique de sinusoïde de fréquence $2 \mathrm{MHz}$; compte-tenu du diamètre du traducteur et de la durée de l'excitation, la distance 35 (resp. 120) mm peut être considérée comme étant "proche" (resp. "éloignée"). Les résultats sont présentés Fig. 2. Les réponses sont quantitativement comparables, par la donnée du rapport d'amplitude exprimé en $\mathrm{dB}$, la référence étant l'amplitude crête-crête de l'écho renvoyé par le disque placé perpendiculairement à $120 \mathrm{~mm}$ du traducteur uniforme. Les résultats expérimentaux du disque sont une partie de résultats publiés ailleurs [6] et ceux du cône sont issus de [7] (obtenus avec le même matériel). 

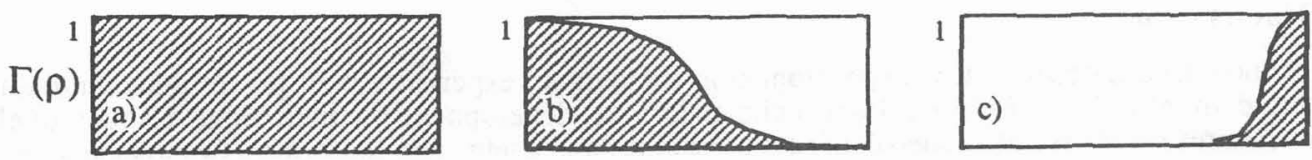

Fig.1. Profil des traducteurs simulés de $19 \mathrm{~mm} \emptyset$. -a) uniforme -b) "à onde plane" -c) "à onde de bord". [Simulated transducers profile of 19-mm-diam. -a) uniform -b) "edge wave"-c) "plane wave" transducers.]
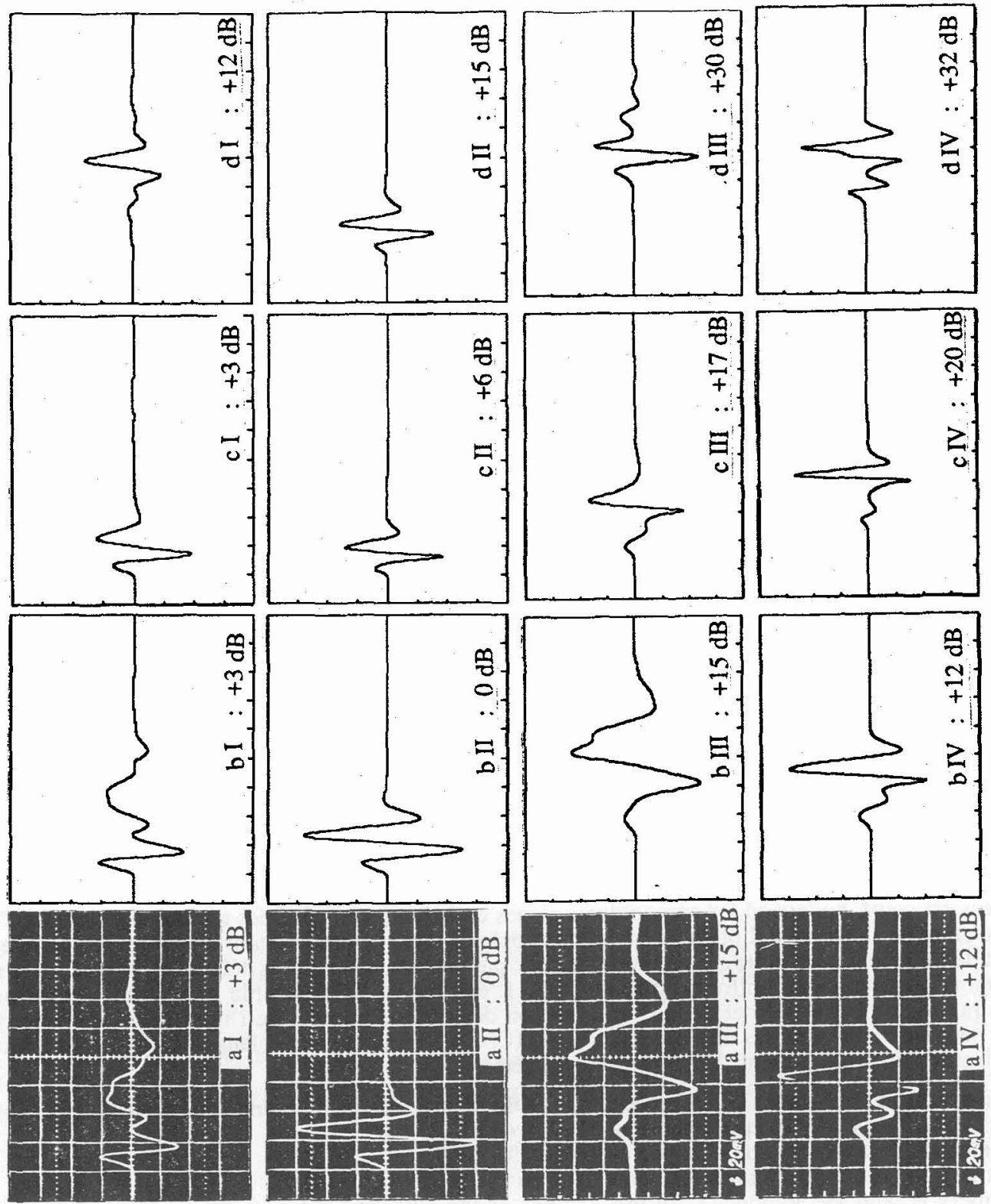

Fig.2. Réponses échographiques expérimentale (a) et simulées pour les traducteurs uniforme (b) TOP (c) et TOB (d). Disque $4 \mathrm{~mm}-\varnothing \perp$ à $35 \mathrm{~mm}$ (I), $\perp$ à $120 \mathrm{~mm}$ (II), cône $150^{\circ} 4 \mathrm{~mm}-\varnothing$ à $35 \mathrm{~mm}$ (III), à $120 \mathrm{~mm}$ (IV). [Measured echo-responses (a) and simulated for uniform (b), plane-wave (c) and edge-wave transducers for a $4 \mathrm{~mm}-\varnothing$ disk target, $\perp$ and at $35 \mathrm{~mm}$ (I) or $120 \mathrm{~mm}$ (II), cone $150^{\circ} 4 \mathrm{~mm}-\emptyset$ at $35 \mathrm{~mm}$ (III) or $120 \mathrm{~mm}$ (IV).] 


\section{Discussion.}

Le très bon accord observé dans la confrontation simulation / expérience pour les résultats du traducteur uniforme montre la validité de l'approche théorique développée; on peut donc risquer quelques commentaires sur les résultats spéculatifs concernant les traducteurs non-uniformes. Comme on avait pu le prévoir, la simplification engendrée par la modification du profil est surtout sensible lorsque la cible est placée près du traducteur.

Pour le disque perpendiculaire à l'axe du traducteur, on s'attend à une structure simple de l'écho; pourtant lorsqu'il est placé à $35 \mathrm{~mm}$ du traducteur uniforme, la forme complexe due à la séparation des contributions plane et de bord peut laisser croire à la présence de trois éléments diffusants distincts. Par ailleurs, le premier écho arrive à l'instant $2 \mathrm{~d} / \mathrm{c}$, où d est la distance traducteur-cible. Avec le TOP, l'écho semble unique et commence au même instant $2 \mathrm{~d} / \mathrm{c}$. Avec le TOB, l'essentiel de l'énergie est portée par un seul écho qui arrive en retard par rapport au temps de trajet le plus court : comme l'énergie provient de et revient au bord du traducteur, le temps de trajet typique est de $2\left(\mathrm{~d}^{2}+\mathrm{a}^{2}\right)^{1 / 2} / \mathrm{c}$ et dépend donc non-linéairement de la distance d. A propos des amplitudes observées, on voit que la correction par rapport à la référence est identique pour le traducteur uniforme et le TOP alors que celle concernant le TOB est plus importante de $9 \mathrm{~dB}$.

Toujours pour le disque mais cette fois placé à $120 \mathrm{~mm}$ du traducteur, on constate que la structure de l'écho pour le traducteur uniforme est plus simple qu'a $35 \mathrm{~mm}$ car la différence de marche entre les contributions planes et de bord est plus courte et ces dernières sont pratiquement confondues. Il est remarquable que la forme des échos obtenus avec les deux traducteurs modifiés est dans ce cas sensiblement la même (comme la cible est petite, on tend progressivement vers un écho qui est la dérivée seconde de v(t) [3]). Cependant, dans le cas des traducteurs modifiés, l'écho est mieux localisé en temps. Une fois encore, l'instant d'arrivée de l'énergie dans le cas de TOB est légèrement en retard par rapport à celui pour les deux autres traducteurs (mais d'autant moins que l'on est loin du traducteur). Les corrections d'amplitudes sont cette fois de $6 \mathrm{~dB}$ dans le cas du TOP et de $15 \mathrm{~dB}$ dans le cas du TOB. Cette différence risque d'être encore plus importante en pratique à ces distances plus longues dans le cas de matériaux acoustiquement absorbant (l'atténuation est négligée dans la modélisation).

Pour le cône, on s'attend cette fois à une réponse composée de deux échos correspondant pour le premier à la diffraction par la pointe du cône puis pour le second à la diffraction par la rupture de pente où le cône est coupé (correspondant à un diamètre de $4 \mathrm{~mm}$ ). Compte-tenu de son angle au sommet et de son diamètre maximal, on peut estimer facilement l'écart temporel entre ces deux échos à environ $0.6 \mu$ s (après allée et retour). Avec le traducteur uniforme, cette structure est à peine visible dans le cas $120 \mathrm{~mm}$ et indécelable pour $35 \mathrm{~mm}$. Avec les traducteurs modifiés, elle apparaît au contraire assez clairement quelle que soit la distance; les mêmes commentaires qu'au paragraphe précédent sur l'amplitude et sur les décalages temporels peuvent être faits ici.

\section{Conclusion.}

Les traducteurs dont on a modifié le profil de source et de sensibilité de manière à privilégier les composantes plane ou de bord rayonnent un champ large bande structurellement plus simple que les traducteurs conventionnels uniformes. En émission-réception (échographie), cette simplification est renforcée car ces effets de diffraction existent également en réception. L'interprétation des échos reçus après diffusion sur une cible en est simplifiée et donc certaines ambiguîtés peuvent être levées. Ce phénomène est d'autant plus vrai que le diffuseur est près du traducteur ou que le signal électrique excitateur transitoire du traducteur est court, i.e. quand les composantes planes et de bord sont séparées temporellement. Le TOP présente en outre l'avantage d'offrir une relation simple (linéaire) entre l'instant d'arrivée de l'écho et la position de l'élément de la cible qui en est à l'origine; par ailleurs, il est moins défavorisé énergétiquement par rapport au traducteur uniforme que le TOB. L'emploi du TOP semble donc très justifié pour l'amélioration de la détection et de la discrimination des défauts proches de l'interface. Le même type d'étude peut être mené pour les traducteurs focalisés (CND) et rectangulaires plans (médical) pour lesquels la modification du profil présente le même genre d'intérêt.

[1] Lhémery A. et De Vadder D., in proceedings of the First French Conference on Acoustics, P. Filippi and M. Zakharia eds., (Editions de physique, 1990), 439-442.

[2] Lhémery A., J. Acoust. Soc. Am. 90 (1991), 2799-2807.

[3] Lhémery A., Thèse de doctorat de l’Université du Maine (France) (1990).

[4] Lhémery A. and De Vadder $D_{*}$, in proceedings of the 12th World Conference on NonDestructive Testing, J. Boogard and G.M. van Dijk eds., (Elsevier, Amsterdam, 1989), 200-202.

[5] Brittain R.H. and Weight J.P., Ultrasonics 25 (1987), 100-106.

[6] Lhémery A., Weight J.P. and Raillon R., to be published in Review of Progress in Quantitative NonDestructive Evaluation, Vol.11, (Plenum Press, N-Y, 1992).

[7] Gatcombe C., PhD Thesis, The City University (London, United Kingdom) (1990). 